Bull. Korean Math. Soc. 51 (2014), No. 1, pp. 237-252

http://dx.doi.org/10.4134/BKMS.2014.51.1.237

\title{
THE HYPONORMAL TOEPLITZ OPERATORS ON THE VECTOR VALUED BERGMAN SPACE
}

\author{
Yufeng Lu, Puyu Cui, and Yanyue Shi
}

\begin{abstract}
In this paper, we give a necessary and sufficient condition for the hyponormality of the block Toeplitz operators $T_{\Phi}$, where $\Phi=F+G^{*}$, $F(z), G(z)$ are some matrix valued polynomials on the vector valued Bergman space $L_{a}^{2}\left(\mathbb{D}, \mathbb{C}^{n}\right)$. We also show some necessary conditions for the hyponormality of $T_{F+G^{*}}$ with $F+G^{*} \in h^{\infty} \otimes M_{n \times n}$ on $L_{a}^{2}\left(\mathbb{D}, \mathbb{C}^{n}\right)$.
\end{abstract}

\section{Introduction}

Let $\mathbb{D}$ and $\mathbb{T}$ be the open unit disk and unit circle in the complex plane $\mathbb{C}$ respectively and $d A$ be the normalized Lebesgue area measure on $\mathbb{D}$. $h^{\infty}$ denotes the space of all bounded harmonic functions on $\mathbb{D} . L^{\infty}(\mathbb{D}, d A)$ and $L^{2}(\mathbb{D}, d A)$ denote the space of essential bounded measurable functions and the space of the square integrable functions on $\mathbb{D}$ with respect to $d A$, respectively. The Bergman space $L_{a}^{2}$ consists of all analytic functions in $L^{2}(\mathbb{D}, d A)$. We denote the space of vector valued square integrable functions on $\mathbb{D}$ by $L^{2}\left(\mathbb{D}, \mathbb{C}^{n}\right)=L^{2}(\mathbb{D}, d A) \otimes \mathbb{C}^{n}$ and the vector valued Bergman space on $\mathbb{D}$ by $L_{a}^{2}\left(\mathbb{D}, \mathbb{C}^{n}\right)=L_{a}^{2} \otimes \mathbb{C}^{n}$, respectively, where $\otimes$ denotes the Hilbert space tensor product. In this paper $F^{T}$ denotes the transpose of the matrix $F$ and $G^{*}$ denotes the adjoint of the matrix $G$.

Let $M_{n \times n}$ be the set of all $n \times n$ complex matrices. The block Toeplitz operator with matrix symbol $\Phi(z)=\left[\varphi_{i j}(z)\right]_{n \times n} \in L^{\infty}(\mathbb{D}, d A) \otimes M_{n \times n}$ (the space of matrix valued essential bounded Lebesgue measurable functions on $\mathbb{D}$ ) is defined by $T_{\Phi} h=P(\Phi h)$ and the block Hankel operator with matrix symbol $\Phi(z)$ is defined by $H_{\Phi} h=(I-P)(\Phi h)$, where $P$ is the orthogonal projection from $L^{2}\left(\mathbb{D}, \mathbb{C}^{n}\right)$ onto $L_{a}^{2}\left(\mathbb{D}, \mathbb{C}^{n}\right)$. If we set $L^{\infty}\left(\mathbb{D}, \mathbb{C}^{n}\right)=L^{\infty}(\mathbb{D}, d A) \oplus \cdots \oplus$ $L^{\infty}(\mathbb{D}, d A)$ and $L_{a}^{2}\left(\mathbb{D}, \mathbb{C}^{n}\right)=L_{a}^{2} \oplus \cdots \oplus L_{a}^{2}$, then the block Toeplitz operator $T_{\Phi}$

Received March 13, 2013.

2010 Mathematics Subject Classification. 47B38.

Key words and phrases. hyponormality, block Toeplitz operator, block Hankel operator.

This research is supported by NSFC No.11271059 and Research Fund for the Doctoral Program of Higher Education of China. 
and the block Hankel operator $H_{\Phi}$ have the following matrix representations :

$$
T_{\Phi}=\left[\begin{array}{ccc}
T_{\varphi_{11}} & \cdots & T_{\varphi_{1 n}} \\
\vdots & & \vdots \\
T_{\varphi_{n 1}} & \cdots & T_{\varphi_{n n}}
\end{array}\right] \text { and } H_{\Phi}=\left[\begin{array}{ccc}
H_{\varphi_{11}} & \cdots & H_{\varphi_{1 n}} \\
\vdots & & \vdots \\
H_{\varphi_{n 1}} & \cdots & H_{\varphi_{n n}}
\end{array}\right]
$$

where

$$
\Phi=\left[\begin{array}{ccc}
\varphi_{11} & \cdots & \varphi_{1 n} \\
\vdots & & \vdots \\
\varphi_{n 1} & \cdots & \varphi_{n n}
\end{array}\right]
$$

A bounded linear operator $A$ on a Hilbert space is called hyponormal if $A^{*} A-A A^{*}$ is a positive operator. There is an extensive literature on hyponormal Toeplitz operators on $H^{2}(\mathbb{T})$ (the Hardy space on $\left.\mathbb{T}\right)[2,4,6,8,12]$. The Bergman space is more complex than the Hardy space. So characterizations of hyponormality of the Toeplitz operators are more difficult, and there are only some results for the Toeplitz operators with certain symbols $[1,7,9,10,11]$. Since $H^{2}(\mathbb{T}) \otimes \mathbb{C}^{n}$ shares a lot of nice properties of $H^{2}(\mathbb{T})$, there is also an elegant characterization of the hyponormality of the Toeplitz operators with the bounded symbols on $H^{2}(\mathbb{T}) \otimes \mathbb{C}^{n}[3,5]$. In this paper, the hyponormality of the block Toeplitz operators with certain symbols on $L_{a}^{2}\left(\mathbb{D}, \mathbb{C}^{n}\right)$ will be discussed.

H. Sadraoui first showed that if $f, g \in H^{\infty}$ (the space of bounded functions on $\mathbb{D}$ ), and $f^{\prime}, g^{\prime} \in H^{2}$ (the space of Hardy space on $\mathbb{D}$ ) such that $T_{f+\bar{g}}$ is hyponormal on $L_{a}^{2}$, then $\left|f^{\prime}(z)\right|>\left|g^{\prime}(z)\right|$ almost everywhere on $\mathbb{T}$ [11]. Later, P. Ahern and Z. Cuckovic [1] generalized H. Sadraoui's result by a mean value inequality and Berezin transform to the following theorem.

Theorem 1.1 ([1]). Suppose that $f, g$ are holomorphic in $\mathbb{D}$, that $\varphi=f+\bar{g}$ is bounded in $\mathbb{D}$ and that $T_{\varphi}$ is hyponormal on $L_{a}^{2}$. Then

$$
\varlimsup_{z \rightarrow \xi}\left(\left|f^{\prime}(z)\right|^{2}-\left|g^{\prime}(z)\right|^{2}\right) \geq 0 \quad \xi \in \mathbb{T} .
$$

In particular, if $f^{\prime}$ and $g^{\prime}$ are continuous at $\xi \in \mathbb{T}$, then $\left|f^{\prime}(\xi)\right| \geq\left|g^{\prime}(\xi)\right|$.

The condition in the above theorem is not sufficient, but for the Toeplitz operators with certain trigonometric polynomial symbols and under some assumptions, the condition is also sufficient:

Theorem $1.2([10])$. Let $\varphi(z)=f(z)+\overline{g(z)}$, where $f(z)=a_{m} z^{m}+a_{n} z^{n}$, $g(z)=b_{m} z^{m}+b_{n} z^{n}(0<m<n)$ with $\left|a_{n}\right| \leq\left|b_{n}\right|$. Then $T_{\varphi}$ is hyponormal on $L_{a}^{2}$ if and only if $\left|f^{\prime}(z)\right| \geq\left|g^{\prime}(z)\right|$ on $\mathbb{T}$.

The hyponormality of $T_{\Psi}$ with $\Psi \in L^{\infty}(\mathbb{T}) \otimes M_{n \times n}$ on $H^{2}(\mathbb{T}) \otimes \mathbb{C}^{n}$ shares the extensions of Cowen's result to $H^{2}(\mathbb{T})$ in $[2,5]$. But the condition of Cowen's Theorem is not sufficiently explicit and is difficult to be verified. There are some explicit conditions of the hyponormal block Toeplitz operators on $H^{2}(\mathbb{T}) \otimes \mathbb{C}^{n}$ under some extremal condition (see the section 4 in [5]). 
In this paper, we consider the hyponormality of $T_{\Phi}$ with $\Phi=F+G^{*} \epsilon$ $h^{\infty} \otimes M_{n \times n}$. In Section 2, we discuss the hyponormality of $T_{\Phi}$ with the matrix with certain polynomials entries symbols. We obtain a necessary and sufficient condition of the hyponormality of $T_{\Phi}$ in Theorem 2.6. In Section 3, we obtain some necessary conditions of the hyponormality of $T_{\Phi}$ on $L_{a}^{2}\left(\mathbb{D}, \mathbb{C}^{n}\right)$ under the assumption $\|F\|_{2}=\|G\|_{2}$. The characterization of normal $T_{\Phi}$ is complicated. We also obtain a simple characterization of normal $T_{\Phi}$ in Theorem 3.5.

\section{Necessary and sufficient condition}

In this section, we will discuss the hyponormality of block Toeplitz operators on $L_{a}^{2}\left(\mathbb{D}, \mathbb{C}^{n}\right)$ with some trigonometric polynomial symbols.

Let $K_{z}(w)=\frac{1}{(1-\bar{z} w)^{2}}(z, w \in \mathbb{D})$ be the reproducing kernel of the Bergman space $L_{a}^{2}$ and $k_{z}(w)=\frac{1-|z|^{2}}{(1-\bar{z} w)^{2}}$ be the normalized reproducing kernel in $L_{a}^{2}$.

First the finite sum of two Hankel operator products on $L_{a}^{2}$ is a positive operator will be discussed. There is the following proposition.

Proposition 2.1. Let $f_{k}=a_{k} z^{M}+b_{k} z^{N}, g_{k}=c_{k} z^{M}+d_{k} z^{N}(0<M<$ $N), 1 \leq k \leq n$. Suppose $\sum_{k=1}^{n}\left|b_{k}\right|^{2} \leq \sum_{k=1}^{n}\left|d_{k}\right|^{2}$. Then $\sum_{k=1}^{n}\left(H_{f_{k}}^{*} H_{\overline{f_{k}}}-\right.$ $\left.H_{\overline{g_{k}}}^{*} H_{\overline{g_{k}}}\right) \geq 0$ if and only if $\sum_{k=1}^{n}\left|f_{k}^{\prime}(z)\right|^{2} \geq \sum_{k=1}^{n}\left|g_{k}^{\prime}(z)\right|^{2}$ on $\mathbb{T}$.

Proof. We first prove the necessity. Since $\sum_{k=1}^{n}\left(H_{f_{k}}^{*} H_{\overline{f_{k}}}-H_{\bar{g}_{k}}^{*} H_{\overline{g_{k}}}\right) \geq 0$, we have

$$
\left\langle\sum_{k=1}^{n}\left(H_{\overline{f_{k}}}^{*} H_{\overline{f_{k}}}-H_{\overline{g_{k}}}^{*} H_{\overline{g_{k}}}\right) k_{z}, k_{z}\right\rangle \geq 0 .
$$

Note that

$$
\begin{aligned}
& \left\langle\sum_{k=1}^{n}\left(H_{\overline{f_{k}}}^{*} H_{\overline{f_{k}}}-H_{\overline{g_{k}}}^{*} H_{\overline{g_{k}}}\right) k_{z}, k_{z}\right\rangle \\
= & \left\langle\sum_{k=1}^{n}\left(\left|f_{k}(z)\right|^{2}-\left|g_{k}(z)\right|^{2}\right) k_{z}, k_{z}\right\rangle-\sum_{k=1}^{n}\left(\left|f_{k}(z)\right|^{2}-\left|g_{k}(z)\right|^{2}\right) .
\end{aligned}
$$

Letting $z \rightarrow \xi \in \mathbb{T}$ and by [1, Theorem 2], we obtain $\sum_{k=1}^{n}\left|f_{k}^{\prime}(z)\right|^{2} \geq$ $\sum_{k=1}^{n}\left|g_{k}^{\prime}(z)\right|^{2}$ on $\mathbb{T}$.

We now prove the sufficiency.

Let $h(z)=\sum_{p=0}^{\infty} \alpha_{p} z^{p} \in L_{a}^{2}$ with $\sum_{p=0}^{\infty} \frac{\left|\alpha_{p}\right|^{2}}{p+1}<\infty$. We have

$$
\begin{aligned}
& \left\langle\sum_{k=1}^{n}\left(H_{\bar{f}_{k}}^{*} H_{\overline{f_{k}}}-H_{\overline{g_{k}}}^{*} H_{\overline{g_{k}}}\right) h, h\right\rangle \\
= & \sum_{p=0}^{\infty} \sum_{q=0}^{\infty} \alpha_{p} \overline{\alpha_{q}}\left\langle\sum_{k=1}^{n}\left(H_{\bar{f}_{k}}^{*} H_{\overline{f_{k}}}-H_{\overline{g_{k}}}^{*} H_{\overline{g_{k}}}\right) z^{p}, z^{q}\right\rangle
\end{aligned}
$$




$$
\begin{aligned}
= & \sum_{p=0}^{\infty}\left|\alpha_{p}\right|^{2}\left\langle\sum_{k=1}^{n}\left(H_{\overline{f_{k}}}^{*} H_{\overline{f_{k}}}-H_{\overline{g_{k}}}^{*} H_{\overline{g_{k}}}\right) z^{p}, z^{p}\right\rangle \\
& +\sum_{p \neq q} \alpha_{p} \overline{\alpha_{q}}\left\langle\sum_{k=1}^{n}\left(H_{\overline{f_{k}}}^{*} H_{\overline{f_{k}}}-H_{\overline{g_{k}}}^{*} H_{\overline{g_{k}}}\right) z^{p}, z^{q}\right\rangle \\
= & \sum_{p=0}^{\infty}\left|\alpha_{p}\right|^{2}\left\langle\sum_{k=1}^{n}\left(H_{\overline{f_{k}}}^{*} H_{\overline{f_{k}}}-H_{\overline{g_{k}}}^{*} H_{\overline{g_{k}}}\right) z^{p}, z^{p}\right\rangle \\
& +\sum_{p=0}^{\infty} 2 \mathcal{R} e\left[\overline{\alpha_{p}} \alpha_{p+N-M} \sum_{k=1}^{n}\left(a_{k} \overline{b_{k}}-c_{k} \overline{d_{k}}\right)\left\langle H_{\bar{z}^{N}} z^{p+N-M}, H_{\bar{z}^{M}} z^{p}\right\rangle\right] \\
\geq & \sum_{p=0}^{\infty}\left|\alpha_{p}\right|^{2}\left\langle\sum_{k=1}^{n}\left(H_{\overline{f_{k}}}^{*} H_{\overline{f_{k}}}-H_{\overline{g_{k}}}^{*} H_{\overline{g_{k}}}\right) z^{p}, z^{p}\right\rangle \\
& -2 \sum_{p=0}^{\infty}\left|\alpha_{p} \alpha_{p+N-M}\right| \cdot\left|\sum_{k=1}^{n}\left(a_{k} \overline{b_{k}}-c_{k} \overline{d_{k}}\right)\right|\left\langle H_{\bar{z}^{N}} z^{p+N-M}, H_{\bar{z}^{M}} z^{p}\right\rangle .
\end{aligned}
$$

Since $\sum_{k=1}^{n}\left|f_{k}^{\prime}(z)\right|^{2} \geq \sum_{k=1}^{n}\left|g_{k}^{\prime}(z)\right|^{2}$ on $\mathbb{T}$, we have

$$
M^{2}\left[\sum_{k=1}^{n}\left(\left|a_{k}\right|^{2}-\left|c_{k}\right|^{2}\right)\right]+N^{2}\left[\sum_{k=1}^{n}\left(\left|b_{k}\right|^{2}-\left|d_{k}\right|^{2}\right)\right] \geq 2 M N\left|\sum_{k=1}^{n}\left(a_{k} \overline{b_{k}}-c_{k} \overline{d_{k}}\right)\right| .
$$

Note that

$$
\begin{aligned}
& \left\langle\sum_{k=1}^{n}\left(H_{\overline{f_{k}}}^{*} H_{\overline{f_{k}}}-H_{\overline{g_{k}}}^{*} H_{\overline{g_{k}}}\right) z^{p}, z^{p}\right\rangle \\
= & \sum_{k=1}^{n}\left(\left|a_{k}\right|^{2}-\left|c_{k}\right|^{2}\right)\left\|H_{\bar{z}^{M}} z^{p}\right\|^{2}+\sum_{k=1}^{n}\left(\left|b_{k}\right|^{2}-\left|d_{k}\right|^{2}\right)\left\|H_{\bar{z}^{N}} z^{p}\right\|^{2} .
\end{aligned}
$$

Denote $\eta^{2}(p)=\left\langle\sum_{k=1}^{n}\left(H_{\overline{f_{k}}}^{*} H_{\overline{f_{k}}}-H_{\overline{g_{k}}}^{*} H_{\overline{g_{k}}}\right) z^{p}, z^{p}\right\rangle$. The hypothesis $\sum_{k=1}^{n}\left|b_{k}\right|^{2} \leq$ $\sum_{k=1}^{n}\left|d_{k}\right|^{2}$ and $\frac{\left\|H_{\bar{z}^{M}} z^{k}\right\|^{2}}{\left\|H_{\bar{z}^{N}} z^{k}\right\|^{2}} \geq \frac{M^{2}}{N^{2}}$ (see [10]) implies that

$$
\begin{aligned}
\eta^{2}(p) & \geq \frac{1}{N^{2}}\left[M^{2} \sum_{k=1}^{n}\left(\left|a_{k}\right|^{2}-\left|c_{k}\right|^{2}\right)+N^{2} \sum_{k=1}^{n}\left(\left|b_{k}\right|^{2}-\left|d_{k}\right|^{2}\right)\right] \cdot\left\|H_{\bar{z}^{N}} z^{p}\right\|^{2} \\
& \geq \frac{2 M N}{N^{2}}\left|\sum_{k=1}^{n}\left(a_{k} \overline{b_{k}}-c_{k} \overline{d_{k}}\right)\right| \cdot\left\|H_{\bar{z}^{N}} z^{p}\right\|^{2}
\end{aligned}
$$

and

$$
\eta^{2}(p) \geq \frac{1}{M^{2}}\left[M^{2} \sum_{k=1}^{n}\left(\left|a_{k}\right|^{2}-\left|c_{k}\right|^{2}\right)+N^{2} \sum_{k=1}^{n}\left(\left|b_{k}\right|^{2}-\left|d_{k}\right|^{2}\right)\right] \cdot\left\|H_{\bar{z}^{M}} z^{p}\right\|^{2}
$$




$$
\geq \frac{2 M N}{M^{2}}\left|\sum_{k=1}^{n}\left(a_{k} \overline{b_{k}}-c_{k} \overline{d_{k}}\right)\right| \cdot\left\|H_{\bar{z}^{M}} z^{p}\right\|^{2} .
$$

Therefore, direct calculation shows that

$$
\begin{aligned}
& \sum_{p=0}^{\infty}\left|\alpha_{p}\right|^{2} \eta^{2}(p) \\
= & \sum_{p=0}^{N-M-1}\left|\alpha_{p}\right|^{2} \eta^{2}(p)+\frac{1}{2} \sum_{p=N-M}^{\infty}\left|\alpha_{p}\right|^{2} \eta^{2}(p)+\frac{1}{2} \sum_{p=N-M}^{\infty}\left|\alpha_{p}\right|^{2} \eta^{2}(p) \\
= & \sum_{p=0}^{N-M-1}\left[\left|\alpha_{p}\right|^{2} \eta^{2}(p)+\frac{1}{2}\left|\alpha_{p+N-M}\right|^{2} \eta^{2}(p+N-M)\right] \\
& +\frac{1}{2} \sum_{p=N-M}^{\infty}\left[\left|\alpha_{p}\right|^{2} \eta^{2}(p)+\left|\alpha_{p+N-M}\right|^{2} \eta^{2}(p+N-M)\right] \\
\geq & \sum_{p=0}^{\infty}\left|\alpha_{p} \alpha_{p+N-M}\right| \cdot \eta(p) \cdot \eta(p+N-M) \\
\geq & \sum_{p=0}^{\infty}\left|\alpha_{p} \alpha_{p+N-M}\right| \cdot\left|\sum_{k=1}^{n}\left(a_{k} \overline{b_{k}}-c_{k} \overline{d_{k}}\right)\right| \cdot\left\|H_{\bar{z}^{M}} z^{p}\right\| \cdot\left\|H_{\bar{z}^{N}} z^{p+N-M}\right\| \\
\geq & \sum_{p=0}^{\infty}\left|\alpha_{p} \alpha_{p+N-M}\right| \cdot\left|\sum_{k=1}^{n}\left(a_{k} \overline{b_{k}}-c_{k} \overline{d_{k}}\right)\right|\left\langle H_{\bar{z}^{N}} z^{p+N-M}, H_{\bar{z}^{M}} z^{p}\right\rangle,
\end{aligned}
$$

which completes the proof.

The following theorem shows that if $T_{\Phi}$, the Toeplitz operator on $L_{a}^{2}\left(D, \mathbb{C}^{n}\right)$ with harmonic symbol $\Phi$, is hyponormal, then $\Phi$ is normal.

Theorem 2.2. Let $\Phi(z)=F(z)+G^{*}(z) \in h^{\infty} \otimes M_{n \times n}$. If $T_{\Phi}$ on $L_{a}^{2}\left(\mathbb{D}, \mathbb{C}^{n}\right)$ is hyponormal, then $\Phi^{*}(z) \Phi(z)=\Phi(z) \Phi^{*}(z)$ almost everywhere on $\mathbb{D}$.

Proof. Denote $T=\left(T_{i j}\right)_{i, j=1}^{n}=T_{\Phi}^{*} T_{\Phi}-T_{\Phi} T_{\Phi}^{*}$, where $T_{i j}=\sum_{p=1}^{n}\left[T_{\overline{\varphi_{p i}}} T_{\varphi_{p j}}-\right.$ $\left.T_{\varphi_{i p}} T_{\overline{\varphi_{j p}}}\right]$. Let $x_{i}=(\underbrace{0, \ldots, 0}_{i-1}, k_{z}, 0, \ldots)^{T}(1 \leq i \leq n)$. Since $T_{\Phi}$ is hyponormal, we get $\left\langle T_{i i} k_{z}, k_{z}\right\rangle \geq 0$. Simple calculation implies that

$$
\left\langle T_{\overline{\varphi_{p i}}} T_{\varphi_{p i}} k_{z}, k_{z}\right\rangle=\left\langle\left|f_{p i}\right|^{2} k_{z}, k_{z}\right\rangle+f_{p i}(z) \overline{g_{p i}(z)}+\overline{g_{p i}(z)} f_{p i}(z)+\left|g_{p i}(z)\right|^{2} .
$$

Denote $z=r \xi, 0<r<1, \xi \in \mathbb{T}$. Letting $r \rightarrow 1$, we have

$$
\begin{aligned}
& \lim _{r \rightarrow 1}\left\langle T_{\overline{\varphi_{p i}}} T_{\varphi_{p i}} k_{z}, k_{z}\right\rangle \\
= & \lim _{r \rightarrow 1}\left\langle\left|f_{p i}\right|^{2} k_{z}, k_{z}\right\rangle+\lim _{r \rightarrow 1}\left[f_{p i}(z) \overline{g_{p i}(z)}+\overline{g_{p i}(z)} f_{p i}(z)+\left|g_{p i}(z)\right|^{2}\right] \\
= & \left|\varphi_{p i}(\zeta)\right|^{2}
\end{aligned}
$$


for almost all $\zeta \in \mathbb{T}$, where the second equality follows from the dominant convergence theorem.

Therefore

$$
\lim _{r \rightarrow 1}\left\langle T_{i i} k_{z}, k_{z}\right\rangle=\sum_{p=1}^{n}\left(\left|\varphi_{p i}(\zeta)\right|^{2}-\left|\varphi_{i p}(\zeta)\right|^{2}\right) \geq 0
$$

for almost all $\zeta \in \mathbb{T}$. Note that

$$
\lim _{r \rightarrow 1} \sum_{i=1}^{k-1}\left\langle T_{i i} k_{z}, k_{z}\right\rangle+\lim _{r \rightarrow 1} \sum_{i=k+1}^{n}\left\langle T_{i i} k_{z}, k_{z}\right\rangle=\sum_{p=1}^{n}\left(\left|\varphi_{k p}(\zeta)\right|^{2}-\left|\varphi_{p k}(\zeta)\right|^{2}\right) \geq 0
$$

and

$$
\lim _{r \rightarrow 1}\left\langle T_{k k} k_{z}, k_{z}\right\rangle=\sum_{p=1}^{n}\left(\left|\varphi_{p k}(\zeta)\right|^{2}-\left|\varphi_{k p}(\zeta)\right|^{2}\right) \geq 0 .
$$

Thus we deduce that

$$
\lim _{r \rightarrow 1}\left\langle T_{k k} k_{z}, k_{z}\right\rangle=\sum_{p=1}^{n}\left(\left|\varphi_{p k}(\zeta)\right|^{2}-\left|\varphi_{k p}(\zeta)\right|^{2}\right)=0
$$

for almost all $\zeta \in \mathbb{T}$ and all $0 \leq k \leq n$.

$$
\begin{aligned}
& \text { Letting } y_{k m}=(\underbrace{0, \ldots, 0}_{k-1}, c k_{z}, \underbrace{0, \ldots, 0}_{m-1}, k_{z}, 0, \ldots)^{T}, c=i,-i, 1,-1 \text {, we get } \\
& \lim _{r \rightarrow 1}\left\langle T_{k} k+m k k_{z}, k_{z}\right\rangle=\sum_{p=1}^{n}\left[\overline{\varphi_{p k}(\zeta)} \varphi_{p(k+m)}(\zeta)-\varphi_{k p}(\zeta) \overline{\varphi_{(k+m) p}(\zeta)}\right]=0
\end{aligned}
$$

for all $1 \leq k \leq n, 1 \leq m \leq n-1$. In conclusion, we have that if $T_{\Phi}$ is hyponormal, then $\Phi^{*}(\zeta) \Phi(\zeta)=\Phi(\zeta) \Phi^{*}(\zeta)$ for almost all $\zeta \in \mathbb{T}$. Taking Poisson integral of the above equality, it follows that $\Phi^{*} \Phi=\Phi \Phi^{*}$ almost everywhere on $\mathbb{D}$ and the proof is complete.

Corollary 2.3. Let $\Phi(z)=F(z)+G^{*}(z) \in h^{\infty} \otimes M_{n \times n}$. If $T_{\Phi}^{*} T_{\Phi}-T_{\Phi} T_{\Phi}^{*}$ is compact on $L_{a}^{2}\left(\mathbb{D}, \mathbb{C}^{n}\right)$, then $\Phi^{*}(z) \Phi(z)=\Phi(z) \Phi^{*}(z)$ almost everywhere on $\mathbb{D}$.

In the rest of this section, we let $\Phi(z)=F(z)+G^{*}(z) \in h^{\infty} \otimes M_{n \times n}$. Then there is the following representation,

$$
\begin{aligned}
T_{\Phi}^{*} T_{\Phi}-T_{\Phi} T_{\Phi}^{*} & =T_{\Phi}^{*} T_{\Phi}-T_{\Phi^{*} \Phi}+\left(T_{\Phi \Phi^{*}}-T_{\Phi} T_{\Phi}^{*}\right)+T_{\Phi^{*} \Phi-\Phi \Phi^{*}} \\
& =-H_{\Phi}^{*} H_{\Phi}+H_{\Phi^{*}}^{*} H_{\Phi^{*}}^{*}+T_{\Phi^{*} \Phi-\Phi \Phi^{*}} \\
& =H_{F^{*}}^{*} H_{F^{*}}-H_{G^{*}}^{*} H_{G^{*}}+T_{\Phi^{*} \Phi-\Phi \Phi^{*}} .
\end{aligned}
$$

By the above equalities and Theorem 2.2, we know that $T_{\Phi}$ is hyponormal if and only if $H_{F^{*}}^{*} H_{F^{*}}-H_{G^{*}}^{*} H_{G^{*}} \geq 0$.

In the rest part of this section, we let $\Phi(z)=F(z)+G^{*}(z)$, where $F(z)=$ $A z^{M}+B z^{N}, G(z)=C z^{M}+D z^{N}(0<M<N), A, B, C, D \in M_{n \times n}$ and there is the following matrix representation,

$$
H_{F^{*}}^{*} H_{F^{*}}-H_{G^{*}}^{*} H_{G^{*}}
$$




$$
=\left[\begin{array}{ccc}
\sum_{k=1}^{n}\left(H_{\overline{f_{1 k}}}^{*} H_{\overline{f_{1 k}}}-H_{\overline{g_{1 k}}}^{*} H_{\overline{g_{1 k}}}\right) & \cdots & \sum_{k=1}^{n}\left(H_{\overline{f_{1 k}}}^{*} H_{\overline{f_{n k}}}-H_{\overline{g_{1 k}}}^{*} H_{\overline{g_{n k}}}\right) \\
\vdots & \vdots \\
\sum_{k=1}^{n}\left(H_{\overline{f_{n k}}}^{*} H_{\overline{f_{1 k}}}-H_{\overline{g_{n k}}}^{*} H_{\overline{g_{1 k}}}\right) & \cdots & \sum_{k=1}^{n}\left(H_{\overline{f_{n k}}}^{*} H_{\overline{f_{n k}}}-H_{\overline{g_{n k}}}^{*} H_{\overline{g_{n k}}}\right)
\end{array}\right] .
$$

Corollary 2.4. Let $F=\operatorname{diag}\left[f_{1}, \ldots, f_{n}\right]$ and $G=\operatorname{diag}\left[g_{1}, \ldots, g_{n}\right]$. Suppose $f_{k}=a_{k} z^{M}+b_{k} z^{N}, g_{k}=c_{k} z^{M}+d_{k} z^{N}(0<M<N)$ with $\left|b_{k}\right| \leq\left|d_{k}\right| 1 \leq k \leq n$. Then $T_{F+G^{*}}$ is hyponormal if and only if $\left|f_{k}^{\prime}(z)\right| \geq\left|g_{k}^{\prime}(z)\right|$ on $\mathbb{T}$ for $1 \leq k \leq n$.

Proof. The proof follows from the above matrix representation and the above proposition.

The following theorem gives a necessary condition for the hyponormal block Toeplitz operators with harmonic symbols.

Theorem 2.5. Suppose $F(z), G(z) \in\left[H^{\infty} \cap C^{1}(\bar{D})\right] \otimes M_{n \times n}$. If $T_{F+G^{*}}$ on $L_{a}^{2}\left(\mathbb{D}, \mathbb{C}^{n}\right)$ is hyponormal, then $F^{\prime}(z)\left[F^{*}(z)\right]^{\prime}-G^{\prime}(z)\left[G^{*}(z)\right]^{\prime}$ is a positive semi-definite matrix for all $z \in \mathbb{T}$.

Proof. Let $x=\left(c_{1} k_{z}, \ldots, c_{n} k_{z}\right)^{T}\left(c_{i} \in \mathbb{C}, 1 \leq i \leq n\right)$. By the precious theorem, we have $\left\langle H_{F^{*}}^{*} H_{F^{*}}-H_{G^{*}}^{*} H_{G^{*}} x, x\right\rangle \geq 0$. Simple calculation implies $\sum_{p=1}^{n} \sum_{q=1}^{n} c_{q} \overline{c_{p}}\left\langle\sum_{k=1}^{n}\left(H_{\overline{f_{p k}}}^{*} H \overline{f_{q k}}-H_{\overline{g_{p k}}}^{*} H_{\overline{g_{q k}}}\right) k_{z}, k_{z}\right\rangle \geq 0$. By [1, Theorem 2], we have $\sum_{p=1}^{n} \sum_{q=1}^{n} c_{q} \overline{c_{p}} \sum_{k=1}^{n}\left[f_{p k}^{\prime}(\xi) \overline{f_{q k}^{\prime}(\xi)}-g_{p k}^{\prime}(\xi) \overline{g_{q k}^{\prime}(\xi)}\right] \geq 0$ on $\mathbb{T}$ and this completes the proof.

The first main result of this paper is the following theorem.

Theorem 2.6. Let $\Phi(z)=F(z)+G^{*}(z), F(z)=A z^{M}+B z^{N}, G(z)=C z^{M}+$ $D z^{N}(0<M<N)$. Suppose that $D D^{*}-B B^{*}$ is a positive semi-definite matrix and $\left|\left\langle\left[B A^{*}-D C^{*}\right] \gamma, \gamma\right\rangle_{\mathbb{C}^{n}} \cdot\left\langle\left[B A^{*}-D C^{*}\right] \delta, \delta\right\rangle_{\mathbb{C}^{n}}\right|^{\frac{1}{2}} \geq\left|\left\langle\left[B A^{*}-D C^{*}\right] \gamma, \delta\right\rangle_{\mathbb{C}^{n}}\right|$, where $A, B, C, D \in M_{n \times n},\langle,\rangle_{\mathbb{C}^{n}}$ is the inner product in $\mathbb{C}^{n}$ and $\gamma, \delta \in \mathbb{C}^{n}$. Then $T_{\Phi}$ is hyponormal if and only if $F^{\prime}(z)\left[F^{*}(z)\right]^{\prime}-G^{\prime}(z)\left[G^{*}(z)\right]^{\prime}$ is a positive semi-definite matrix for all $z \in \mathbb{T}$.

Proof. Without loss of generality, we only need to proof the sufficiency for $n=2$.

Let $y=\left(h_{1}, h_{2}\right)^{T}$, where $h_{1}(z)=\sum_{l=0}^{\infty} \alpha_{l} z^{l}$ with $\sum_{l=0}^{\infty}\left|\alpha_{l}\right|^{2} /(l+1)<$ $\infty, h_{2}(z)=\sum_{l=1}^{\infty} \beta_{l} z^{l}$ with $\sum_{l=0}^{\infty}\left|\beta_{l}\right|^{2} /(l+1)<\infty$. By [10, Lemma 2.1], $\left\{H_{\bar{z}^{M}}\left(z^{k}\right)\right\}_{k=0}^{\infty}$ are pairwise orthogonal in $L^{2}(D, d A)$ and $\left\{H_{\bar{z}^{k}}\left(z^{M}\right)\right\}_{k=0}^{\infty}$ are also pairwise orthogonal in $L^{2}(D, d A)$, so

$$
\begin{aligned}
& \left\langle H_{F^{*}}^{*} H_{F^{*}}-H_{G^{*}}^{*} H_{G^{*}} y, y\right\rangle \\
= & \sum_{k=0}^{\infty}\left\{\left|\alpha_{k}\right|^{2} \sum_{l=1}^{2}\left(\left|a_{1 l}\right|^{2}-\left|c_{1 l}\right|^{2}\right)+\left|\beta_{k}\right|^{2} \sum_{l=1}^{2}\left(\left|a_{2 l}\right|^{2}-\left|c_{2 l}\right|^{2}\right)\right.
\end{aligned}
$$




$$
\begin{aligned}
& \left.+2 \mathcal{R} e\left[\alpha_{k} \overline{\beta_{k}} \sum_{l=1}^{2}\left(\bar{a}_{2 l} a_{1 l}-\bar{c}_{2 l} c_{1 l}\right)\right]\right\} \cdot\left\|H_{\bar{z}^{M}} z^{k}\right\|^{2} \\
& +\sum_{k=0}^{\infty}\left\{\left|\alpha_{k}\right|^{2} \sum_{l=1}^{2}\left(\left|b_{1 l}\right|^{2}-\left|d_{1 l}\right|^{2}\right)+\left|\beta_{k}\right|^{2} \sum_{l=1}^{2}\left(\left|b_{2 l}\right|^{2}-\left|d_{2 l}\right|^{2}\right)\right. \\
& \left.+2 \mathcal{R} e\left[\alpha_{k} \overline{\beta_{k}} \sum_{l=1}^{2}\left(\bar{b}_{2 l} b_{1 l}-\bar{d}_{2 l} d_{1 l}\right)\right]\right\} \\
& +2 \sum_{k=0}^{\infty} \mathcal{R} e\left\{\alpha_{k} \bar{\alpha}_{k+N-M} \sum_{l=1}^{2}\left(\bar{a}_{1 l} b_{1 l}-\bar{c}_{1 l} d_{1 l}\right)\right. \\
& +\beta_{k} \bar{\beta}_{k+N-M} \sum_{l=1}^{2}\left(\bar{a}_{2 l} b_{2 l}-\bar{c}_{2 l} d_{2 l}\right) \\
& +\alpha_{k} \bar{\beta}_{k+N-M} \sum_{l=1}^{2}\left(\bar{a}_{1 l} b_{2 l}-\bar{c}_{1 l} d_{2 l}\right) \\
& \left.+\beta_{k} \bar{\alpha}_{k+N-M} \sum_{l=1}^{2}\left(\bar{a}_{2 l} b_{1 l}-\bar{c}_{2 l} d_{1 l}\right)\right\}\left\langle H_{\bar{z}^{M}} z^{k}, H_{\bar{z}^{N}} z^{k+N-M}\right\rangle \\
& =\sum_{k=0}^{\infty} \sum_{l=1}^{2}\left(\left|\alpha_{k} a_{1 l}+\beta_{k} a_{2 l}\right|^{2}-\left|\alpha_{k} c_{1 l}+\beta_{k} c_{2 l}\right|^{2}\right) \cdot\left\|H_{\bar{z}^{M}} z^{k}\right\|^{2} \\
& +\sum_{k=0}^{\infty} \sum_{l=1}^{2}\left(\left|\alpha_{k} b_{1 l}+\beta_{k} b_{2 l}\right|^{2}-\left|\alpha_{k} d_{1 l}+\beta_{k} d_{2 l}\right|^{2}\right) \cdot\left\|H_{\bar{z}^{N}} z^{k}\right\|^{2} \\
& +2 \sum_{k=0}^{\infty} \mathcal{R} e\left\{\sum _ { l = 1 } ^ { 2 } \left[\left(\alpha_{k} a_{1 l}+\beta_{k} a_{2 l}\right) \overline{\left(\alpha_{k+N-M} b_{1 l}+\beta_{k+N-M} b_{2 l}\right)}\right.\right. \\
& \left.\left.-\left(\alpha_{k} c_{1 l}+\beta_{k} c_{2 l}\right) \overline{\left(\alpha_{k+N-M} d_{1 l}+\beta_{k+N-M} d_{2 l}\right)}\right]\right\}\left\langle H_{\bar{z}^{M}} z^{k}, H_{\bar{z}^{N}} z^{k+N-M}\right\rangle .
\end{aligned}
$$

By the hypothesis, $F^{\prime}(z)\left[F^{*}(z)\right]^{\prime}-G^{\prime}(z)\left[G^{*}(z)\right]^{\prime}$ is a positive semi-definite matrix for all $z \in \mathbb{T}$, that means, for any $e_{1}, e_{2} \in \mathbb{C}, \sum_{i, j=1}^{2} E_{i j}(z) e_{j} \overline{e_{i}} \geq 0$ on $\mathbb{T}$, where $E_{i, j}=\sum_{p=1}^{2} f_{i p}^{\prime} \overline{f_{j p}^{\prime}}-g_{i p}^{\prime} \overline{g_{j p}^{\prime}}$. It follows that

$$
\sum_{i, j=1}^{2} E_{i j}(z) e_{j} \overline{e_{i}}=\sum_{p=1}^{2}\left(\left|e_{1} f_{1 p}^{\prime}+e_{2} f_{2 p}^{\prime}\right|^{2}-\left|e_{1} g_{1 p}^{\prime}+e_{2} g_{2 p}^{\prime}\right|^{2}\right) \geq 0 .
$$

Simple calculation implies that $F^{\prime}(z)\left[F^{*}(z)\right]^{\prime}-G^{\prime}(z)\left[G^{*}(z)\right]^{\prime}$ is positive semidefinite on $\mathbb{T}$ if and only if

$$
\left[M^{2} \sum_{p=1}^{2}\left(\left|e_{1} a_{1 p}+e_{2} a_{2 p}\right|^{2}-\left|e_{1} c_{1 p}+e_{2} c_{2 p}\right|^{2}\right)\right.
$$




$$
\begin{aligned}
& \left.+N^{2} \sum_{p=1}^{2}\left(\left|e_{1} b_{1 p}+e_{2} b_{2 p}\right|^{2}-\left|e_{1} d_{1 p}+e_{2} d_{2 p}\right|^{2}\right)\right] \\
\geq & \left.2 M N \mid \sum_{p=1}^{2}\left[\left(e_{1} a_{1 p}+e_{2} a_{2 p}\right) \overline{\left(e_{1} b_{1 p}+e_{2} b_{2 p}\right.}\right)-\left(e_{1} c_{1 p}+e_{2} c_{2 p}\right) \overline{\left(e_{1} d_{1 p}+e_{2} d_{2 p}\right)}\right] \mid .
\end{aligned}
$$

Denote

$$
\begin{aligned}
\eta_{k}^{2}= & \sum_{l=1}^{2}\left(\left|\alpha_{k} a_{1 l}+\beta_{k} a_{2 l}\right|^{2}-\left|\alpha_{k} c_{1 l}+\beta_{k} c_{2 l}\right|^{2}\right) \cdot\left\|H_{\bar{z}^{M}} z^{k}\right\|^{2} \\
& +\sum_{l=1}^{2}\left(\left|\alpha_{k} b_{1 l}+\beta_{k} b_{2 l}\right|^{2}-\left|\alpha_{k} d_{1 l}+\beta_{k} d_{2 l}\right|^{2}\right) \cdot\left\|H_{\bar{z}^{N}} z^{k}\right\|^{2} .
\end{aligned}
$$

Since $D D^{*}-B B^{*}$ is positive semi-definite and $\frac{\left\|H_{z_{M}} z^{k}\right\|^{2}}{\left\|H_{\bar{z}^{N}} z^{k}\right\|^{2}} \geq \frac{M^{2}}{N^{2}}$, we have

$$
\begin{aligned}
\frac{\eta_{k}^{2}}{\left\|H_{\bar{z}^{M}} z^{k}\right\|^{2}} \geq & \frac{1}{M^{2}}\left\{M^{2} \sum_{l=1}^{2}\left(\left|\alpha_{k} a_{1 l}+\beta_{k} a_{2 l}\right|^{2}-\left|\alpha_{k} c_{1 l}+\beta_{k} c_{2 l}\right|^{2}\right)\right. \\
& \left.+N^{2} \sum_{l=1}^{2}\left(\left|\alpha_{k} b_{1 l}+\beta_{k} b_{2 l}\right|^{2}-\left|\alpha_{k} d_{1 l}+\beta_{k} d_{2 l}\right|^{2}\right)\right\} \\
\geq & \frac{2 M N}{M^{2}} \mid \sum_{l=1}^{2}\left\{\left(\alpha_{k} a_{1 l}+\beta_{k} a_{2 l}\right) \overline{\left(\alpha_{k} b_{1 l}+\beta_{k} b_{2 l}\right)}\right. \\
& \left.-\left(\alpha_{k} c_{1 l}+\beta_{k} c_{2 l}\right) \overline{\left(\alpha_{k} d_{1 l}+\beta_{k} d_{2 l}\right)}\right\} \mid .
\end{aligned}
$$

Similarly, we have

$$
\begin{aligned}
\frac{\eta_{k}^{2}}{\left\|H_{\bar{z}^{N}} z^{k}\right\|^{2}} \geq \frac{2 M N}{N^{2}} \mid \sum_{l=1}^{2}\{ & \left(\alpha_{k} a_{1 l}+\beta_{k} a_{2 l}\right) \overline{\left(\alpha_{k} b_{1 l}+\beta_{k} b_{2 l}\right)} \\
& \left.-\left(\alpha_{k} c_{1 l}+\beta_{k} c_{2 l}\right) \overline{\left(\alpha_{k} d_{1 l}+\beta_{k} d_{2 l}\right)}\right\} \mid .
\end{aligned}
$$

Denote $\gamma_{k}=\left(\overline{\alpha_{k}}, \overline{\beta_{k}}\right)^{T}$ and $\delta_{k}=\left(\overline{\alpha_{k+N-M}}, \overline{\beta_{k+N-M}}\right)^{T}$. We have the following inequality

$$
\begin{aligned}
& \eta_{k} \eta_{k+N-M} \\
\geq 2 \mid \sum_{l=1}^{2}\left\{\left(\alpha_{k} a_{1 l}+\beta_{k} a_{2 l}\right) \overline{\left(\alpha_{k} b_{1 l}+\beta_{k} b_{2 l}\right)}\right. & \left.\quad-\left(\alpha_{k} c_{1 l}+\beta_{k} c_{2 l}\right) \overline{\left(\alpha_{k} d_{1 l}+\beta_{k} d_{2 l}\right)}\right\}\left.\right|^{\frac{1}{2}} \\
& \cdot \mid \sum_{l=1}^{2}\left\{\left(\alpha_{k+N-M} a_{1 l}+\beta_{k+N-M} a_{2 l}\right) \overline{\left(\alpha_{k+N-M} b_{1 l}+\beta_{k+N-M} b_{2 l}\right)}\right. \\
- & \left.\left(\alpha_{k+N-M} c_{1 l}+\beta_{k+N-M} c_{2 l}\right) \overline{\left(\alpha_{k+N-M} d_{1 l}+\beta_{k+N-M} d_{2 l}\right)}\right\}\left.\right|^{\frac{1}{2}}
\end{aligned}
$$




$$
\begin{aligned}
& \left\langle H_{\bar{z}^{M}} z^{k}, H_{\bar{z}^{N}} z^{k+N-M}\right\rangle \\
= & 2\left|\left\langle A^{*} \gamma_{k}, B^{*} \gamma_{k}\right\rangle_{\mathbb{C}^{2}}-\left\langle C^{*} \gamma_{k}, D^{*} \gamma_{k}\right\rangle_{\mathbb{C}^{2}}\right|^{\frac{1}{2}} \\
& \cdot\left|\left\langle A^{*} \delta_{k}, B^{*} \delta_{k}\right\rangle_{\mathbb{C}^{2}}-\left\langle C^{*} \delta_{k}, D^{*} \delta_{k}\right\rangle_{\mathbb{C}^{2}}\right|^{\frac{1}{2}}\left\langle H_{\bar{z}^{M}} z^{k}, H_{\bar{z}^{N}} z^{k+N-M}\right\rangle .
\end{aligned}
$$

Since $\left|\left\langle\left[B A^{*}-D C^{*}\right] \gamma_{k}, \gamma_{k}\right\rangle_{\mathbb{C}^{2}} \cdot\left\langle\left[B A^{*}-D C^{*}\right] \delta_{k}, \delta_{k}\right\rangle_{\mathbb{C}^{2}}\right|^{\frac{1}{2}} \geq \mid\left\langle\left[B A^{*}-\right.\right.$ $\left.\left.D C^{*}\right] \gamma_{k}, \delta_{k}\right\rangle_{\mathbb{C}^{2}} \mid$, we get $\left|\left\langle A^{*} \gamma_{k}, B^{*} \gamma_{k}\right\rangle_{\mathbb{C}^{2}}-\left\langle C^{*} \gamma_{k}, D^{*} \gamma_{k}\right\rangle_{\mathbb{C}^{2}}\right|^{\frac{1}{2}} \cdot \mid\left\langle A^{*} \delta_{k}, B^{*} \delta_{k}\right\rangle_{\mathbb{C}^{2}}-$ $\left.\left\langle C^{*} \delta_{k}, D^{*} \delta_{k}\right\rangle_{\mathbb{C}^{2}}\right|^{\frac{1}{2}} \geq\left|\left\langle A^{*} \gamma_{k}, B^{*} \delta_{k}\right\rangle_{\mathbb{C}^{2}}-\left\langle C^{*} \gamma_{k}, D^{*} \delta_{k}\right\rangle_{\mathbb{C}^{2}}\right|$. So

$\eta_{k} \eta_{k+N-M}$

$$
\begin{aligned}
\geq 2 & \mid \sum_{l=1}^{2}\left[\left(\alpha_{k} a_{1 l}+\beta_{k} a_{2 l}\right) \overline{\left(\alpha_{k+N-M} b_{1 l}+\beta_{k+N-M} b_{2 l}\right)}\right. \\
& \left.-\left(\alpha_{k} c_{1 l}+\beta_{k} c_{2 l}\right) \overline{\left(\alpha_{k+N-M} d_{1 l}+\beta_{k+N-M} d_{2 l}\right)}\right] \mid\left\langle H_{\bar{z}^{M}} z^{k}, H_{\bar{z}^{N}} z^{k+N-M}\right\rangle .
\end{aligned}
$$

Thus

$$
\begin{aligned}
\sum_{k=0}^{\infty} \eta_{k}^{2} & =\sum_{k=0}^{N-M+1} \eta_{k}^{2}+\sum_{k=0}^{N-M-1} \eta_{k+N-M}^{2}+\sum_{k=2 N-2 M}^{\infty} \eta_{k}^{2} \\
& =\sum_{k=0}^{N-M+1} \eta_{k}^{2}+\frac{1}{2} \sum_{k=0}^{N-M-1} \eta_{k+N-M}^{2}+\frac{1}{2} \sum_{k=N-M}^{\infty} \eta_{k}^{2}+\frac{1}{2} \sum_{k=N-M}^{\infty} \eta_{k+N-M}^{2} \\
& \geq \sum_{k=0}^{\infty} \eta_{k} \eta_{k+N-M} .
\end{aligned}
$$

This completes the proof.

Remark 2.7. The assumption that $D D^{*}-B B^{*}$ is positive semi-definite can not be removed. Let $F, G$ be the diagonal matrices with the matrix entries as $f$ and $g$ in [10, Remark 2.8] and we get the counterexample.

\section{Necessary conditions with $\|F\|_{2}=\|G\|_{2}$}

For a matrix valued analytic function $M \in L^{2}(D, d A) \otimes M_{n \times n}$, let

$$
M(z)=\left[m_{i j}(z)\right]_{n \times n}=\sum_{k=0}^{\infty} M_{k} z^{k}
$$

be the Taylor expansion of $M(z)$. The 2-norm of $M$ is defined by

$$
\|M\|_{2}^{2}=\int_{D} \operatorname{tr}\left[M^{*}(z) M(z)\right] d A(z)=\sum_{k=0}^{\infty} \frac{\operatorname{tr}\left[M_{k}^{*} M_{k}\right]}{k+1},
$$

where $t r$ is the trace of matrix $M$.

Theorem 3.1. Let $\Phi(z)=F(z)+\Phi(0)+G^{*}(z) \in h^{\infty}(D, d A) \otimes M_{n \times n}$ with $\|F\|_{2}=\|G\|_{2}$. If $T_{\Phi}$ is hyponormal, then $P\left(\operatorname{tr}\left[F^{*} F-F F^{*}\right](z)\right)=0$. 
Proof. Let $h_{p}=(\underbrace{0, \ldots, 0}_{p-1}, z^{k}+c, 0, \ldots)^{T}, p \geq 1, k \geq 0$ and $c \in \mathbb{C}$. Since $T_{\Phi}$ is hyponormal, then we have $\left\langle T_{\Phi}^{*} T_{\Phi}-T_{\Phi} T_{\Phi}^{*} h_{p}, h_{p}\right\rangle \geq 0$, and hence

$$
\sum_{p=1}^{n}\left\langle H_{F^{*}}^{*} H_{F^{*}}-H_{G^{*}}^{*} H_{G^{*}} h_{p}, h_{p}\right\rangle=\sum_{p=1}^{n}\left(\left\|H_{F^{*}} h_{p}\right\|^{2}-\left\|H_{G^{*}} h_{p}\right\|^{2}\right) \geq 0 .
$$

Note that

$$
\left\|H_{F^{*}} h_{p}\right\|^{2}=\sum_{q=1}^{n}\left\|H_{\bar{f}_{p q}} z^{k}\right\|^{2}+|c|^{2} \sum_{q=1}^{n}\left\|\bar{f}_{p q}\right\|^{2}+\sum_{q=1}^{n} 2 \mathcal{R} e\left\langle c \bar{f}_{p q}, H_{\bar{f}_{p q}} z^{k}\right\rangle .
$$

By the hypothesis, we arrive

$$
\sum_{p=1}^{n} \sum_{q=1}^{n}\left\|f_{p q}\right\|^{2}=\sum_{p=1}^{n} \sum_{q=1}^{n}\left\|g_{p q}\right\|^{2}
$$

Therefore,

$$
\begin{aligned}
& \sum_{p=1}^{n} \sum_{q=1}^{n}\left(\left\|H_{\bar{f}_{p q}} z^{k}\right\|^{2}-\left\|H_{\bar{g}_{p q}} z^{k}\right\|^{2}\right) \\
& +\sum_{p=1}^{n} \sum_{q=1}^{n} 2 \mathcal{R} e\left[c\left\langle\bar{f}_{p q}, H_{\bar{f}_{p q}} z^{k}\right\rangle-c\left\langle\bar{g}_{p q}, H_{\bar{g}_{p q}} z^{k}\right\rangle\right] \\
= & \sum_{p=1}^{n} \sum_{q=1}^{n}\left(\left\|H_{\bar{f}_{p q}} z^{k}\right\|^{2}-\left\|H_{\bar{p}_{p q}} z^{k}\right\|^{2}\right)+\sum_{p=1}^{n} \sum_{q=1}^{n} 2 \mathcal{R} e\left[c\left\langle\left.\bar{f}_{p q}\right|^{2}-\left|\bar{g}_{p q}\right|^{2}, z^{k}\right\rangle\right] \geq 0 .
\end{aligned}
$$

Letting $|c| \rightarrow \infty$, we get $P\left(\sum_{p=1}^{n} \sum_{q=1}^{n}\left(\left|f_{p q}\right|^{2}-\left|g_{p q}\right|^{2}\right)\right)=0$, that is $P\left(\operatorname{tr}\left[F^{*} F-\right.\right.$ $\left.\left.G G^{*}\right](z)\right)=0$.

Corollary 3.2. Let $F(z)=\sum_{k=1}^{I} F_{k} z^{k}, G(z)=\sum_{k=1}^{I} G_{k} z^{k}$ with $\|F\|_{2}=$ $\|G\|_{2}$. Suppose $T_{F+G^{*}}$ is hyponormal on $L_{a}^{2}\left(D, \mathbb{C}^{n}\right)$. Then

$$
\nu=\left[\begin{array}{cccc}
N_{1,1} & N_{2,2} & \cdots & N_{I, I} \\
0 & N_{1,2} & \cdots & N_{I-1, I} \\
\vdots & 0 & \ddots & \vdots \\
0 & \cdots & 0 & N_{1, I}
\end{array}\right]\left[\begin{array}{c}
\frac{1}{2} \\
\frac{1}{3} \\
\vdots \\
\frac{1}{I+1}
\end{array}\right]=0,
$$

where $N_{l, k}=\operatorname{tr}\left[F_{k}^{*} F_{l}-G_{k}^{*} G_{l}\right], l, k=1,2, \ldots, I$.

Proof. By Theorem 3.1, it is sufficient to show that if $\|F\|_{2}=\|G\|_{2}$, then the vector $\nu=0$ if and only if $P\left(\operatorname{tr}\left[F^{*}(z) F(z)-G(z) G^{*}(z)\right]\right)=0$.

Note that

$$
\begin{aligned}
& \left\langle P\left(\operatorname{tr}\left[F^{*}(z) F(z)-G(z) G^{*}(z)\right]\right), K_{w}\right\rangle \\
= & \left\langle\operatorname{tr}\left[F^{*} F-G G^{*}\right], K_{w}\right\rangle
\end{aligned}
$$




$$
\begin{aligned}
& =\left\langle\operatorname{tr}\left[\sum_{k=1}^{I} F_{k}^{*} \bar{z}^{k} \sum_{l=1}^{I} F_{l} z^{l}-\sum_{k=1}^{I} G_{k}^{*} \bar{z}^{k} \sum_{l=1}^{I} G_{l} z^{l}\right], K_{w}\right\rangle \\
& =\left\langle\operatorname{tr}\left[\sum_{k=1}^{I} \sum_{l=1}^{I}\left(F_{k}^{*} F_{l}-G_{k}^{*} G_{l}\right)\right] \bar{z}^{k} z^{l}, K_{w}\right\rangle \\
& =\operatorname{tr}\left[\sum_{k=1}^{I} \sum_{l=1}^{I}\left(F_{k}^{*} F_{l}-G_{k}^{*} G_{l}\right)\right]\left\langle\bar{z}^{k} z^{l}, K_{w}\right\rangle .
\end{aligned}
$$

By the hypothesis, we know that $\sum_{l \geq k} \frac{\operatorname{tr}\left[F_{k}^{*} F_{l}-G_{k}^{*} G_{l}\right]}{k+1}=0$, that is, $\sum_{l \geq k} \frac{N_{l, k}}{k+1}=$ 0 .

Lemma 3.3. Let $F(z)=\left[f_{i j}\right]_{n \times n}, G(z)=\left[g_{i j}\right]_{n \times n} \in\left[H^{\infty} \cap C^{1}(\bar{D})\right] \otimes M_{n \times n}$. Suppose $\sum_{q=1}^{n}\left(\left|f_{p q}^{\prime}(\xi)\right|^{2}-\left|g_{p q}^{\prime}(\xi)\right|^{2}\right)=0(1 \leq p \leq n)$ for $\xi \in \mathbb{T}$. If $T_{F+G^{*}}$ is hyponormal, then $F^{\prime}(\xi)\left(F^{*}\right)^{\prime}(\xi)=G^{\prime}(\xi)\left(G^{*}\right)^{\prime}(\xi)$ for $\xi \in \mathbb{T}$.

Proof. Let $x_{k}=(\underbrace{0, \ldots, 0}_{k-1}, k_{z}, c k_{z}, 0, \ldots)^{T}(c \in \mathbb{C}), k \geq 1$.

Since $H_{F^{*}}^{*} H_{F^{*}}-H_{G^{*}}^{*} H_{G^{*}} \geq 0$, it follows that

$$
\begin{aligned}
& \left\langle\sum_{p=1}^{n}\left(H_{\bar{f}_{k p}}^{*} H_{\bar{f}_{k p}}-H_{\bar{g}_{k p}}^{*} H_{\bar{g}_{k p}}\right) k_{z}, k_{z}\right\rangle \\
& +2 \mathcal{R} e \bar{c}\left\langle\sum_{p=1}^{n}\left(H_{\bar{f}_{(k+1) p}}^{*} H_{\bar{f}_{k p}}-H_{\bar{g}_{(k+1) p}}^{*} H_{\bar{g}_{k p}}\right) k_{z}, k_{z}\right\rangle \\
& +\left\langle\sum_{p=1}^{n}\left(H_{\bar{f}_{(k+1) p}^{*}}^{*} H_{\bar{f}_{(k+1) p}}-H_{\bar{g}_{(k+1) p}}^{*} H_{\bar{g}_{(k+1) p}}\right) k_{z}, k_{z}\right\rangle \geq 0 .
\end{aligned}
$$

By [1, Theorem 2], we have

$$
\begin{aligned}
& \sum_{p=1}^{n}\left(\left|f_{k p}^{\prime}(\xi)\right|^{2}-\left|g_{k p}^{\prime}(\xi)\right|^{2}\right) \\
& +2 \mathcal{R} e \bar{c}\left[\sum_{p=1}^{n}\left(f_{(k+1) p}^{\prime}(\xi) f_{k p}^{\prime}(\xi)-g_{(k+1) p}^{\prime}(\xi) g_{k p}^{\prime}(\xi)\right)\right] \\
& +\sum_{p=1}^{n}\left(\left|f_{(k+1) p}^{\prime}(\xi)\right|^{2}-\left|g_{(k+1) p}^{\prime}(\xi)\right|^{2}\right) \geq 0 \quad \text { for } \xi \in \mathbb{T} .
\end{aligned}
$$

It follows that $2 \mathcal{R} e \bar{c}\left[\sum_{p=1}^{n}\left(f_{(k+1) p}^{\prime}(\xi) f_{k p}^{\prime}(\xi)-g_{(k+1) p}^{\prime}(\xi) g_{k p}^{\prime}(\xi)\right)\right] \geq 0$.

Taking $c=1,-1, i,-i$, it implies

$$
\sum_{p=1}^{n}\left(f_{(k+1) p}^{\prime}(\xi) f_{k p}^{\prime}(\xi)-g_{(k+1) p}^{\prime}(\xi) g_{k p}^{\prime}(\xi)\right)=0
$$


for $\xi \in \mathbb{T}$. Similarly, letting $y_{k, m}=(\underbrace{0, \ldots, 0}_{k-1}, k_{z}, \underbrace{0, \ldots, 0}_{m-1}, c k_{z}, 0, \ldots)^{T}, k \geq$ $1, m \geq 1$, we have $F^{\prime}(\xi)\left(F^{*}\right)^{\prime}(\xi)=G^{\prime}(\xi)\left(G^{*}\right)^{\prime}(\xi)$ for $\xi \in \mathbb{T}$.

The second main result of this paper is the following theorem.

Theorem 3.4. Let $F+G^{*} \in h^{\infty} \otimes M_{n \times n}$ with $F(0)=G(0)=0_{n \times n}$. Suppose $\|F\|_{2}=\|G\|_{2}$ and $F^{\prime}, G^{\prime} \in H^{2} \otimes M_{n \times n}$. If $T_{F+G^{*}}$ is hyponormal, then

(1) $F(z) F^{*}(z)=G(z) G^{*}(z)$ almost everywhere on $\mathbb{D}$, and

(2) $F^{*}(z) F(z)=G^{*}(z) G(z)$ almost everywhere on $\mathbb{D}$.

Proof. (1) Let $x_{p}=(\underbrace{0, \ldots, 0}_{p-1}, 1,0, \ldots)^{T}, p \geq 1$. Since $T_{F+G^{*}}$ is hyponormal, it follows that $\sum_{q=1}^{n}\left\|f_{p q}\right\|^{2} \geq \sum_{q=1}^{n}\left\|g_{p q}\right\|^{2}$. Note that $\sum_{p=1}^{n} \sum_{q=1}^{n}\left\|f_{p q}\right\|^{2}=$ $\sum_{p=1}^{n} \sum_{q=1}^{n}\left\|g_{p q}\right\|^{2}$. Hence $\sum_{q=1}^{n}\left\|f_{p q}\right\|^{2}=\sum_{q=1}^{n}\left\|g_{p q}\right\|^{2}$.

The following familiar Green's identity is from [1],

$$
\int_{\mathbb{D}} u(z) d A-u(0)=\int_{\mathbb{D}} \Delta u(z) K(z) d A(z)
$$

where $u(z) \in C^{2}(\mathbb{D}) \cap L^{1}(\mathbb{D}, d A), K(z)=\log \frac{1}{|z|^{2}}-\left(1-|z|^{2}\right) \geq 0$ for $z \in \mathbb{D}$. Direct calculation implies that

$$
\begin{aligned}
0 & =\sum_{q=1}^{n}\left\|f_{p q}\right\|^{2}-\sum_{q=1}^{n}\left\|g_{p q}\right\|^{2} \\
& =\int_{\mathbb{D}}\left(\sum_{q=1}^{n}\left|f_{p q}\right|^{2}-\sum_{q=1}^{n}\left|g_{p q}\right|^{2}\right) d A \\
& =\left[\sum_{q=1}^{n}\left(\left|f_{p q}\right|^{2}-\left|g_{p q}\right|^{2}\right)\right](0)+\int_{\mathbb{D}} \Delta\left[\sum_{q=1}^{n}\left(\left|f_{p q}\right|^{2}-\left|g_{p q}\right|^{2}\right)(z)\right] K(z) d A(z) \\
& =0+\int_{\mathbb{D}}\left[\sum_{q=1}^{n}\left(\left|f_{p q}^{\prime}\right|^{2}-\left|g_{p q}^{\prime}\right|^{2}\right)(z)\right] K(z) d A(z) .
\end{aligned}
$$

Let $y_{p}=(\underbrace{0, \ldots, 0}_{p-1}, k_{z}, 0, \ldots)^{T}, p \geq 1$. Since $T_{F+G^{*}}$ is hyponormal, by $[1$,

Theorem 2], it follows that $\varlimsup_{z \rightarrow \xi \in \mathbb{T}} \sum_{q=1}^{n}\left[\left|f_{p q}^{\prime}\right|^{2}-\left|g_{p q}^{\prime}\right|^{2}\right](z) \geq 0$.

$$
\begin{aligned}
& \varlimsup_{z \rightarrow \xi \in \mathbb{T}} \sum_{q=1}^{n}\left[\left|f_{p q}^{\prime}\right|^{2}-\left|g_{p q}^{\prime}\right|^{2}\right](z), \\
= & \varlimsup_{z \rightarrow \xi \in \mathbb{T}} \lim _{r \rightarrow 1} \sum_{q=1}^{n}\left[\left|\left(f_{p q}^{\prime}\right)^{r}\right|^{2}-\left|\left(g_{p q}^{\prime}\right)^{r}\right|^{2}\right](z) \\
= & \lim _{r \rightarrow 1} \varlimsup_{z \rightarrow \xi \in \mathbb{T}} \sum_{q=1}^{n}\left[\left|\left(f_{p q}^{\prime}\right)^{r}\right|^{2}-\left|\left(g_{p q}^{\prime}\right)^{r}\right|^{2}\right](z)
\end{aligned}
$$




$$
\begin{aligned}
& =\lim _{r \rightarrow 1} \sum_{q=1}^{n}\left[\left|\left(f_{p q}^{\prime}\right)^{r}\right|^{2}-\left|\left(g_{p q}^{\prime}\right)^{r}\right|^{2}\right](\xi) \\
& =\sum_{q=1}^{n}\left[\left|f_{p q}^{\prime}\right|^{2}-\left|g_{p q}^{\prime}\right|^{2}\right](\xi) \quad \text { for almost all } \quad \xi \in \mathbb{T},
\end{aligned}
$$

where $f^{r}(z)=f(r z)(0<r<1)$ for all $z \in \mathbb{D}$. Taking the Poisson integral of the above equality, it follows that $\sum_{q=1}^{n}\left(\left|f_{p q}^{\prime}\right|^{2}-\left|g_{p q}^{\prime}\right|^{2}\right)(z) \geq 0$, this implies that $\sum_{q=1}^{n}\left(\left|f_{p q}^{\prime}\right|^{2}-\left|g_{p q}^{\prime}\right|^{2}\right)(z)=0$ for almost all $z \in \mathbb{D}$. Denote $f_{p q}^{\prime}(z)=\sum_{k=1}^{\infty} a_{k}^{(p q)} z^{k}$ and $\widehat{f_{p q}^{\prime}}(z)=\sum_{k=1}^{\infty} \overline{a_{k}^{(p q)}} z^{k}$. The above equality is equal to

$$
\sum_{q=1}^{n} f_{p q}^{\prime}(z) \widehat{f_{p q}^{\prime}}(\bar{z})-\sum_{q=1}^{n} g_{p q}^{\prime}(z) \widehat{g_{p q}^{\prime}}(\bar{z})=0 \quad \text { a.e. on } \mathbb{D} \text {. }
$$

It follows from a well-known result in several complex variables (that is, if $f(z, w)$ is holomorphic in $\mathbb{D} \times \mathbb{D}$ and $f(z, \bar{z})=0$, then $f(z, w) \equiv 0$ for $(z, w) \in$ $\mathbb{D} \times \mathbb{D})$, then

$$
\sum_{q=1}^{n} f_{p q}^{\prime}(z) \widehat{f_{p q}^{\prime}}(w)-\sum_{q=1}^{n} g_{p q}^{\prime}(z) \widehat{g_{p q}^{\prime}}(w)=0 \quad \text { a.e. on } \mathbb{D} \times \mathbb{D} .
$$

So

$$
\begin{aligned}
0 & =\int_{0}^{z} \sum_{q=1}^{n} f_{p q}^{\prime}(u) \widehat{f_{p q}^{\prime}}(v)-\sum_{q=1}^{n} g_{p q}^{\prime}(u) \widehat{g_{p q}^{\prime}}(v) d u \\
& =\sum_{q=1}^{n} f_{p q}(z) \widehat{f_{p q}^{\prime}}(v)-\sum_{q=1}^{n} g_{p q}(z) \widehat{g_{p q}^{\prime}}(v) \\
& =\int_{0}^{w} \sum_{q=1}^{n} f_{p q}(z) \widehat{f_{p q}^{\prime}}(v)-\sum_{q=1}^{n} g_{p q}(z) \widehat{g_{p q}^{\prime}}(v) d v \\
& =\sum_{q=1}^{n} f_{p q}(z) \widehat{f_{p q}}(w)-\sum_{q=1}^{n} g_{p q}(z) \widehat{g_{p q}}(w) .
\end{aligned}
$$

Thus $\sum_{q=1}^{n}\left|f_{p q}\right|^{2}(z)=\sum_{q=1}^{n}\left|g_{p q}\right|^{2}(z)(p \geq 1)$ for almost all $z \in \mathbb{D}$. By Lemma 3.3 and the previous proof, we get $F(z) F^{*}(z)=G(z) G^{*}(z)$ for almost all $z \in \mathbb{D}$.

(2) Note that $\left(F+G^{*}\right)\left(F^{*}+G\right)=\left(F^{*}+G\right)\left(F+G^{*}\right)$. Taking Laplace transform $\Delta$ of the above equality, we get $F^{\prime}(z) F^{\prime *}(z)-G^{\prime}(z) G^{\prime *}(z)=F^{\prime *}(z) F^{\prime}(z)-$ $G^{\prime *}(z) G^{\prime}(z)$. Using a similar argument as in the proof of (1), one can prove the conclusion.

The following corollary generalizes [10, Theorem 3.3].

Theorem 3.5. Let $F+G^{*} \in h^{\infty} \otimes M_{n \times n}$ with $F(0)=G(0)=0_{n \times n}$. Suppose $\|F\|_{2}=\|G\|_{2}, F^{\prime}, G^{\prime} \in H^{2} \otimes M_{n \times n}$ and $\operatorname{det} F$, $\operatorname{det} G$ are not identically zero. Then the following statements are equivalent: 
(1) $T_{F+G^{*}}$ on $L_{a}^{2}\left(D, \mathbb{C}^{n}\right)$ is hyponormal,

(2) $T_{F+G^{*}}$ on $L_{a}^{2}\left(D, \mathbb{C}^{n}\right)$ is normal,

(3) there exists a constant unitary matrix $U$ such that $F(z)=G(z) U$.

Proof. $(2) \Rightarrow(1)$ is trivial.

$(3) \Rightarrow(2)$. Since

$$
\begin{aligned}
H_{F^{*}}^{*} H_{F^{*}}-H_{G^{*}}^{*} H_{G^{*}} & =H_{(G U)^{*}}^{*} H_{(G U) *}-H_{G^{*}}^{*} H_{G^{*}} \\
& =H_{U^{*} G^{*}}^{*} H_{U^{*} G^{*}}-H_{G^{*}}^{*} H_{G^{*}} \\
& =H_{G^{*}}^{*}\left(U^{*}\right)^{*} H_{U^{*} G^{*}}-H_{G^{*}}^{*} H_{G^{*}} \\
& =H_{G^{*}}^{*} U U^{*} H_{G^{*}}-H_{G^{*}}^{*} H_{G^{*}}=0,
\end{aligned}
$$

we get $T_{F+G^{*}}$ is normal.

$(1) \Rightarrow(3)$. By Theorem 3.4, it follows that $F(z) F^{*}(z)=G(z) G^{*}(z)$ for almost all $z \in \mathbb{D}$. Since $\operatorname{det} F, \operatorname{det} G$ are not identically zero analytic functions, we have $G^{-1}(z) F(z)=G^{*}(z)\left(F^{*}\right)^{-1}(z)$. Note that $G^{-1}(z) F(z)$ is analytic and $G^{*}(z)\left(F^{*}\right)^{-1}(z)$ is co-analytic. So there exists a constant matrix $U$ such that $G^{-1}(z) F(z)=G^{*}(z)\left(F^{*}\right)^{-1}(z)=U$. Therefore $F(z)=\left(U F^{*}(z)\right)^{*} U=$ $F(z) U^{*} U$ and $G^{*}(z)=U(G(z) U)^{*}=U U^{*} G^{*}(z)$. Hence $U$ is a unitary matrix and hence $F(z)=G(z) U$.

\section{References}

[1] P. Ahern and Z. Cuckovic, A mean value inequality with applications to Bergman space operators, Pacific J. Math. 173 (1996), no. 2, 295-305.

[2] C. Cowen, Hyponormality of Toeplitz operators, Proc. Amer. Math. Soc. 103 (1988), no. 3, 809-812.

[3] R. E. Curto, I. S. Hwang, and W. Y. Lee, Hyponormality and subnormality of block Toeplitz operators, Adv. Math. 230 (2012), no. 4-6, 2094-2151.

[4] D. R. Farenick and W. Y. Lee, Hyponormality and spectra of Toeplitz operators, Trans. Amer. Math. Soc. 348 (1996), no. 10, 4153-4174.

[5] C. Gu, J. Hendricks, and D. Rutherford, Hyponormality of block Toeplitz operators, Pacific J. Math. 223 (2006), no. 1, 95-111.

[6] C. Gu and J. E. Shapiro, Kernels of Hankel operators and hyponormality of Toeplitz operators, Math. Ann. 319 (2001), no. 3, 553-572.

[7] I. S. Hwang, Hyponormal Toeplitz operators on the Bergman space, J. Korean Math. Soc. 42 (2005), no. 2, 387-403.

[8] I. S. Hwang and W. Y. Lee, Hyponormality of trigonometric Toeplitz operators, Trans. Amer. Math. Soc. 354 (2002), no. 6, 2461-2474.

[9] I. S. Hwang and J. Lee, Hyponormal Toeplitz operators on the Bergman space. II, Bull. Korean Math. Soc. 44 (2007), no. 3, 517-522.

[10] Y. F. Lu and Y. Y. Shi, Hyponormal Toeplitz operators on the weighted Bergman space, Integral Equations Operator Theory 65 (2009), 115-129.

[11] H. Sadraoui, Hyponormality of Toeplitz operators and composition operators, Thesis, Purdue University, 1992.

[12] K. H. Zhu, Hyponormal Toeplitz operators with polynomial symbols, Integral Equations Operator Theory 21 (1995), no. 3, 376-381. 
YUFENG LU

School of Mathematical Sciences

Dalian University of TECHNOLOGY

Dalian 116024, P. R. China

E-mail address: lyfdlut@dlut.edu.cn

Puyu Cui

School of Mathematical Sciences

Dalian University of Technology

DAlian 116024, P. R. CHINA

E-mail address: cuipuyu1234@sina.com

YANYUE SHI

School of Mathematical Sciences

OCEAN University OF CHINA

QingdaO, 266000, P. R. China

E-mail address: shiyanyue@gmail.com 